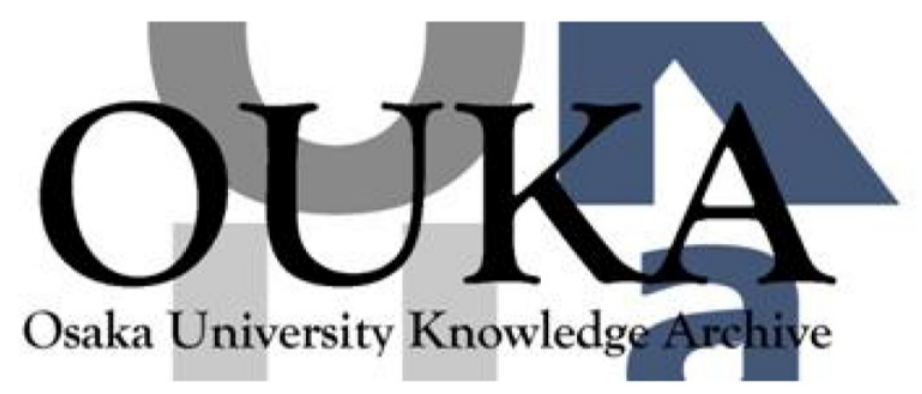

\begin{tabular}{|c|l|}
\hline Title & $\begin{array}{l}\text { Autoinjection of electrons into a wake field } \\
\text { using a capillary with attached cone }\end{array}$ \\
\hline Author(s) & Mori, Y.; Sentoku, Y.; Kondo, K. et al. \\
\hline Citation & $\begin{array}{l}\text { Physics of Plasmas. 16(12) p. 123103-1-p. 123103- } \\
6\end{array}$ \\
\hline Issue Date & $2009-12$ \\
\hline oaire:version & VoR \\
\hline URL & https://hdl. handle. net/11094/3355 \\
\hline rights & \\
\hline Note & \\
\hline
\end{tabular}

Osaka University Knowledge Archive : OUKA

https://ir. Library. osaka-u. ac. jp/

Osaka University 


\title{
Autoinjection of electrons into a wake field using a capillary with attached cone
}

\author{
Y. Mori, ${ }^{1, a)}$ Y. Sentoku, ${ }^{2}$ K. Kondo, ${ }^{3,4, b)}$ K. Tsuji, ${ }^{3,4}$ N. Nakanii, ${ }^{3,4}$ S. Fukumochi, ${ }^{3,4}$ \\ M. Kashihara, ${ }^{3,4}$ K. Kimura, ${ }^{3,4}$ K. Takeda, ${ }^{3}$ K. A. Tanaka, ${ }^{3,4}$ T. Norimatsu, ${ }^{3,4}$ \\ Tsuyoshi Tanimoto, ${ }^{3,4}$ H. Nakamura, ${ }^{3,4}$ M. Tampo, ${ }^{3, b)}$ R. Kodama, ${ }^{3,4}$ E. Miura, ${ }^{5}$ K. Mima, ${ }^{3}$ \\ and Y. Kitagawa ${ }^{1}$ \\ ${ }^{1}$ The Graduate School for the Creation of New Photonics, 1955-1 Kurematsu-cho, Hamamatsu, \\ Shizuoka 431-1202, Japan \\ ${ }^{2}$ Department of Physics, University of Nevada, 5625 Fox Avenue, Reno, Nevada 89506, USA \\ ${ }^{3}$ Institute of Laser Engineering, Osaka University, 2-6 Yamada-oka, Suita, Osaka 565-0871, Japan \\ ${ }^{4}$ Graduate School of Engineering, Osaka University, 2-6 Yamada-oka, Suita, Osaka 565-0871, Japan \\ ${ }^{5}$ National Institute of Advanced Industrial Science and Technology, AIST Tsukuba Central 2, \\ 1-1-1 Umezono, Tsukuba, Ibaraki 305-8568, Japan
}

(Received 27 February 2009; accepted 12 November 2009; published online 8 December 2009)

\begin{abstract}
By using a cone attached to a capillary, electrons generated through a laser interaction were autoinjected and accelerated in a low-density wake field. The cone attached to the entrance of the capillary serves as an electron supplier. It increases the number of electrons from below the detection limit to $1.1 \mathrm{pC}$ and the energy from 4 to $30 \mathrm{MeV}$. A two-dimensional particle-in-cell simulation reveals that a significant number of energetic electrons are extracted from the surface of the cone and are subsequently trapped in the wake field and accelerated in the capillary. (c) 2009 American Institute of Physics. [doi:10.1063/1.3271152]
\end{abstract}

\section{INTRODUCTION}

In a laser electron accelerator, ${ }^{1}$ the advantage of using a capillary target to extend the acceleration distance over a Rayleigh length was first reported by Kitagawa $e t a l .{ }^{2}$ The resulting acceleration length was $1 \mathrm{~cm}$, and the electron energy gain was up to $100 \mathrm{MeV}$. This was the first evidence that a capillary-based electron accelerator holds promise for laser-plasma electron acceleration. Nowadays, in relation to beam quality optimization, ${ }^{3-7}$ using a 3 -cm-long capillary discharge waveguide, the energy gain is as much as $1 \mathrm{GeV}$ for a monoenergetic beam. ${ }^{8,9}$

Capillary-based electron acceleration is thus a key component of high-energy laser electron accelerators. Such a capillary can extend the laser interaction over a Rayleigh length so as to increase the acceleration. This extension of the acceleration length is essential to raise the energy gain. According to theory, it requires an intense laser guided in a plasma of low density. In Ref. 1, the maximum electron energy was $W_{\max }=e E_{L}^{\mathrm{cr}} l_{a}^{\mathrm{dp}} \simeq 2 m_{e} c^{2}\left(\omega / \omega_{p}\right)^{2}$ where $e E_{L}^{\mathrm{cr}} \cong m_{e} c \omega_{p}$ is the critical longitudinal field in the wave-breaking limit and $l_{a}^{\mathrm{dp}} \cong 2 \omega^{2} c / \omega_{p}^{3}$ is the dephasing length in which the electrons enter a deceleration phase. Here $m_{e}, c, \omega$, and $\omega_{p}$ denote the electron mass, speed of light, laser frequency, and plasma frequency, respectively. For example, to achieve $1 \mathrm{GeV}$ energy gain with a laser wavelength of $1 \mu \mathrm{m}$ requires $l_{a}^{\mathrm{dp}}$ to be $0.8 \mathrm{~cm}$. Increasing the gain to $10 \mathrm{GeV}$ extends the length to $25 \mathrm{~cm}$.

An electron obtains energy from a capillary plasma waveguide according to the following sequence: (i) a laser is

\footnotetext{
${ }^{a}$ Electronic mail: ymori@gpi.ac.jp.

${ }^{b}$ Present address: Kansai Photon Science Institute, Japan Atomic Energy Agency.
}

guided into the capillary, (ii) a wake field excitation is produced, (iii) an electron is injected, and (iv) the electron is accelerated. In 2004, using a cone-attached capillary target, we confirmed laser guiding and wake excitation, resulting in electron acceleration of up to $100 \mathrm{MeV}^{2}$ A gold cone glued to the entrance of the capillary guided the laser in. A prepulse discharged a capillary wall, providing a plasma in the capillary, and subsequently a main pulse excited the wake field. However, the mechanism of electron injection was not discussed. Electron injection into a wake field has been a key issue for laser electron acceleration ever since the first demonstration of this phenomenon. ${ }^{10,11}$ Achieving controlled acceleration requires that the wake field driven in the plasma trap only the electrons that are injected from an external source rather than those from the plasma. The latter is called self-trapping. It is particularly important for realizing a multistaged acceleration. Historically, injection has been accomplished with an external rf accelerator. ${ }^{11,12}$ The idea of using an additional pulse has also been proposed. ${ }^{13-16}$ Recently, optical injection has provided controlled electron injection and acceleration for a gas jet target. ${ }^{17}$ Nevertheless, for capillary acceleration, a suitable injection mechanism has not yet been discovered. We need to clarify the mechanism to achieve staged acceleration of a capillary-based accelerator with promise of multi-GeV gain and compact size.

In the present article, the mechanism of automatic electron injection into a capillary-sustained wake field is studied. Two different structures are used: (i) a cone-attached capillary and (ii) a bore capillary without a cone. In previous experiments, in the absence of a guiding cone, an intense laser beam could not be focused into a capillary of $100 \mu \mathrm{m}$ or smaller bore size. To guide the light into the capillary without a cone, the laser beam pointing accuracy was im- 


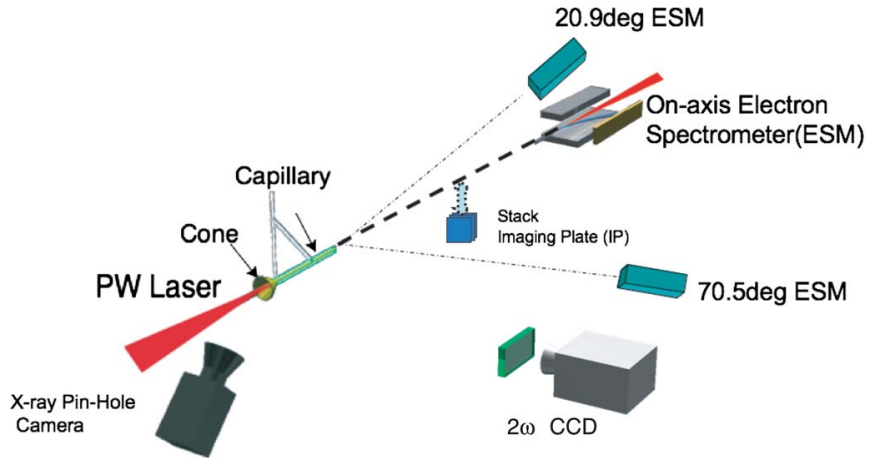

FIG. 1. (Color online) Experimental setup for cone capillary electron acceleration.

proved by accounting for the thermal lens, and a large bore capillary was employed. Since the present experiment uses a prepulse-free laser, a preplasma discharge from a capillary wall does not occur. Therefore, gas was introduced into the target chamber to ensure that the laser discharges inside the capillary. The laser irradiation in the capillary exhibits electron bunch generation of at most $2.7 \mathrm{MeV}$ with a normalized emittance of $1.6 \pi \mathrm{mm}$ mrad. This energy is far lower than the $30 \mathrm{MeV}$ obtained with a cone capillary. The experiment and a particle-in-cell (PIC) simulation reveal that the cone attached to the capillary plays an important role in the automatic injection of electrons into the wake field. Because a reduction in the electron density leads to an increase in the phase velocity of the plasma wake field, high-speed electrons have to be trapped. Prior to the acceleration, an intense laser pulse must kick off electrons into the wake potential. ${ }^{18} \mathrm{~A}$ cone-attached capillary is an alternative scheme to accomplish both automatic injection of the electrons and an extension of the acceleration length using a moderate laser pulse.

This article presents techniques for injecting high-energy electrons into a capillary and for accelerating the electrons. A hollow cone is attached to the entrance of the capillary target. In Sec. II, the experimental setup and results are presented. In Sec. III, the wake amplitude in the capillary and the electron trapping threshold are analyzed. In Sec. IV, a two-dimensional PIC simulation demonstrates the generation of electrons through an interaction between the laser and the cone and the trapping of those electrons in the wake field sustained in the capillary. Section V discusses the results, and Sec. VI gives the conclusions.

\section{EXPERIMENTAL SETUP AND RESULTS}

We used the prepulse-free petawatt laser system at the Institute of Laser Engineering (ILE) ${ }^{19}$ A $1.054 \mu \mathrm{m}$ laser pulse [pulse energy of $80 \mathrm{~J}$ and full width at half maximum (FWHM) of $800 \mathrm{fs}]$ was focused onto the entrance of a cone capillary using a $f / 7.6$ off-axis parabolic mirror to produce a focal spot size of $80 \mu \mathrm{m}$ FWHM, providing an intensity of $2 \times 10^{18} \mathrm{~W} / \mathrm{cm}^{2}$ corresponding to $a_{0}=1.2$. The Rayleigh length was $4.8 \mathrm{~mm}$.

The experimental setup is shown in Fig. 1. An electron spectrometer (ESM) was mounted along the laser axis at a distance of $1.3 \mathrm{~m}$ from the center of the target chamber. A
$1.0 \mathrm{~T}$ permanent magnet installed in the ESM analyzes the electron energies by determining their trajectories using a detector array composed of an imaging plate (IP), Fuji Film BAS-SR $2025{ }^{20}$ The ESM provides energy resolution $\delta E / E$ of 0.07 for $100 \mathrm{MeV}$ electron detection and 0.03 for $10 \mathrm{MeV}$ electron detection, respectively, with an 8-mm-diameter collimator. IPs were placed on both sides of the beam axis in order to measure the energy distribution of electrons to the right of the axis and of ions to the left.

Laser light transmitted through the rear window of an ESM was first attenuated by diffusers or neutral density filters and then guided to a spectrometer in order to detect sidebands. A charge coupled device camera collected a side image of the $2 \omega(527 \mathrm{~nm})$ plasma emission along the capillary, confirming that the laser goes through it.

In order to monitor the spatial divergence of the accelerated electrons, we set up two ESMs (Ref. 21) at angles of $20.9^{\circ}$ and $70.5^{\circ}$ relative to the axis. A stack of IPs $400 \mathrm{~mm}$ away from the target measured the angular distribution. The IP is covered with $\mathrm{Cu}$ and $\mathrm{Al}$ filters and uses CR-39 and radiochromic film to measure the proton angular distribution. The minimum detectable energy of the electrons is $0.6 \mathrm{MeV}$ through the filters.

The laser system has been changed from previous experiments. ${ }^{2}$ To begin with, we have improved the laser beam pointing stability. An off-axis parabolic mirror was aligned to give a pointing stability of $\pm 20 \mu \mathrm{m}$. An x-ray pinhole camera monitored whether the laser spot appears in the entrance aperture of the cone capillary. Second, the laser pulse characteristics have been altered. In previous experiments, we had used a prepulse laser, ${ }^{22}$ with leading pulses 0.7 and $6.3 \mathrm{~ns}$ prior to the main pulse, having intensities of about $2 \times 10^{-3}$ of that of the main pulse. The prepulses ablated the capillary wall, producing a plasma of number density of $6.2 \times 10^{16} \mathrm{~cm}^{-3}{ }^{2}$. The present experiment used a prepulse-free laser, which had a foot pulse originating from the optical parametric generation, with an intensity of $1.5 \times 10^{-8}$ that of the main pulse. Since the foot pulse intensity is $\sim 10^{10} \mathrm{~W} / \mathrm{cm}^{2}$, too weak to ablate the solid surface, we filled the target chamber with gas to ensure discharge inside the capillary. The target chamber was filled with nitrogen gas to a pressure of 100 mTorr. Above $I=2.0 \times 10^{18} \mathrm{~W} / \mathrm{cm}^{2}$, nitrogen is ionized to $\mathrm{N}^{5+}$ by barrier suppression ionization, ${ }^{23}$ resulting in an electron density of approximately $10^{16} \mathrm{~cm}^{-3}$. In that case the plasma wavelength $\lambda_{p}$ is comparable to the laser pulse propagation length $\tau c \sim 240 \mu \mathrm{m}$.

To clarify the effect on the electron acceleration of attaching the hollow cone to the capillary, we measured the electron energy distribution for two target structures: (i) the cone capillary and (ii) the capillary without a cone, as sketched in Fig. 2(a). The hollow cone was made of gold with a length of $1 \mathrm{~mm}$, a conical angle of $30^{\circ}$, an entrance aperture of $500 \mu \mathrm{m}$, and an exit diameter of $150 \mu \mathrm{m}$ to fit the capillary. The capillary was made of glass and had an internal diameter of $150 \mu \mathrm{m}$, an external diameter of about $190 \mu \mathrm{m}$, and a length of $2 \mathrm{~cm}$. In Fig. 2(b), IP images for both targets indicate that a relativistic electron beam was detected when the cone capillary was used. The horizontal 


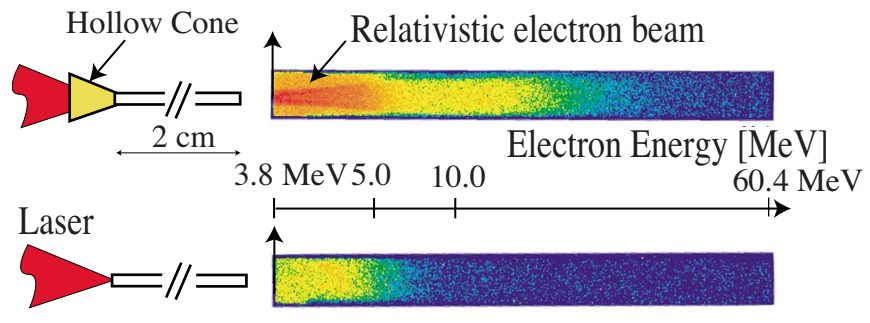

(a)

(b)

FIG. 2. (Color online) (a) Hollow cone capillary (upper sketch) and capillary without cone (lower sketch). (b) Corresponding IP images from the ESM for the two targets.

axis represents the electron energy from 3.8 to $60.4 \mathrm{MeV}$, while the vertical axis represents the beam size collimated by the aperture. The beam exhibits defocusing due to the magnet. By contrast, no beam component was observed when the capillary without cone was used. The bright area from 3.8 to $5 \mathrm{MeV}$ is the background due to $\gamma$-rays from the beam collimation apertures. Backgrounds were also detected on the positive-charge deflecting side. The capillary without cone did not generate energetic electrons for two reasons. First, the laser may not focus into the capillary. Second, even if the laser does focus into the capillary, there may not be electrons with energies exceeding the minimum detection limit of the ESM. The first explanation is ruled out by the lack of $\gamma$-ray signals detected by the x-ray camera located at the rear of the target. When the laser beam loses pointing and hits the capillary, the camera detects strong $\gamma$-ray signals. The side image of $2 \omega$ emission further confirmed that the laser travels through the capillary as demonstrated in Fig. 3 by the bright spot at the exit of the capillary.

The IP stack measurements confirm the existence of an electron bunch with a normalized emittance of $1.7 \pi \mathrm{mm}$ mrad at $0.6 \mathrm{MeV}$. We conclude that the capillary without cone generated an electron bunch but that its energy was below $4 \mathrm{MeV}$, the detection limit of the ESM.
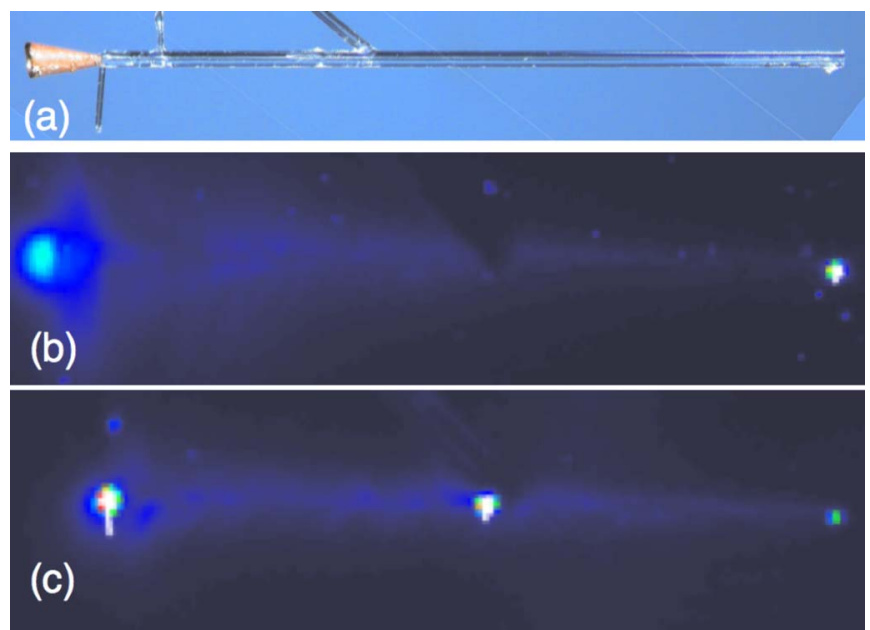

FIG. 3. (Color online) (a) Photograph of the cone capillary (side view). $2 \omega$ $(527 \mathrm{~nm})$ emission along the capillary axis for (b) the cone capillary and (c) the capillary without cone. The left and right spots correspond to the entrance and exit points, respectively. The middle bright spot in (c) corresponds to a glue point for a post supporting the capillary.

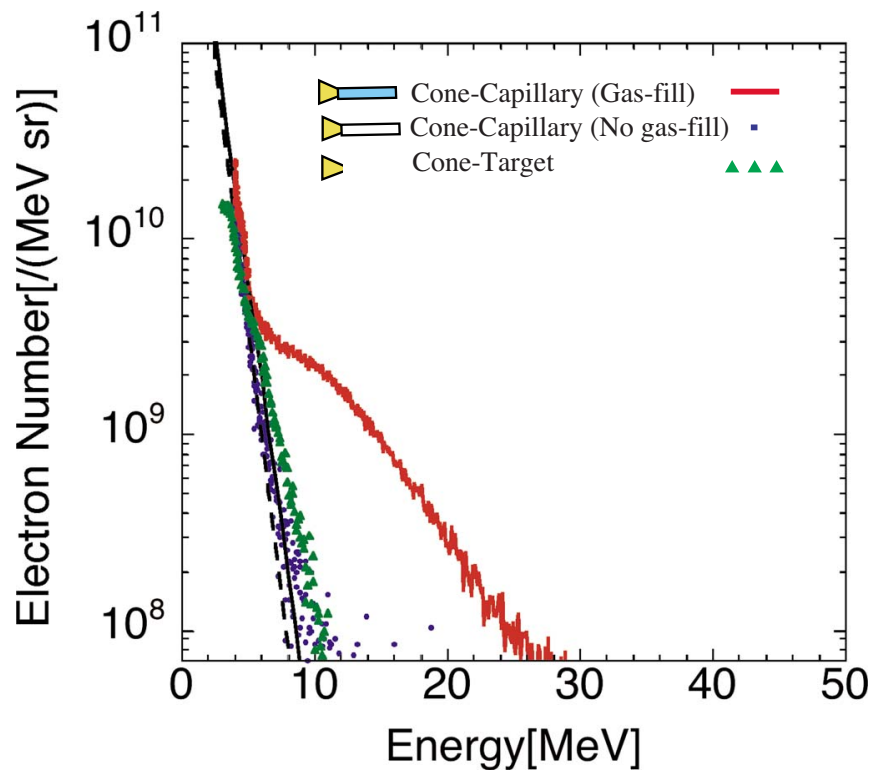

FIG. 4. (Color online) Electron energy spectra for the cone capillary with and without ambient gas, as well as for a hollow cone. The thin solid line and the dashed line are Maxwellian fits.

We compared the electron energy distribution of the cone capillary with and without gas to that of a simple hollow cone in Fig. 4. Since background $\mathrm{x}$ rays radiate symmetrically onto the IP detector of the ESM, the signals were integrated vertically along the detector plate, and the background was subtracted from the integrated signals on the positive-charge side. Figure 4 shows that the cone capillary containing ambient gas generated electrons of up to $30 \mathrm{MeV}$, while the hollow cone generated electrons only up to $10 \mathrm{MeV}$. Comparison of them indicates that the electrons extracted from the cone are automatically accelerated in the capillary filled with ambient gas. In the absence of gas, a portion of the capillary does not accelerate electrons. We conclude that electrons with energies below $10 \mathrm{MeV}$ originate from the cone structure. From Fig. 2, measurement of the electron divergence as $9 \mathrm{mrad}$ enables us to estimate the total charge of the accelerated electrons having energies between 5 and $30 \mathrm{MeV}$. At $10 \mathrm{MeV}$, the cone capillary with gas had $2 \times 10^{9}$ electrons $/ \mathrm{MeV} / \mathrm{sr}$, while the one without gas only had $10^{8}$ electrons $/ \mathrm{MeV} / \mathrm{sr}$. Therefore the coneproduced $0.2 \mathrm{pC}$ of electrons between 5 and $10 \mathrm{MeV}$. The capillary in the ambient gas increased them to $0.7 \mathrm{pC}$ between 5 and $10 \mathrm{MeV}$ and $0.4 \mathrm{pC}$ between 10 and $30 \mathrm{MeV}$. Since both energy spectra have Maxwellian distributions, the total number of electrons might be determined by the lower energy electrons, $N_{0} / T_{e} \exp \left[-E / T_{e}\right]$ below $5 \mathrm{MeV}$ where $E$ is electron energy, $N_{0}$ is the total number of electrons, and $T_{e}$ is the electron temperature. Without the ambient gas, this expression predicts $1.7 \times 10^{12}$ electrons/sr, nearly the same as $1.6 \times 10^{12}$ electrons/sr calculated with the gas. These results show that the ambient gas does not increase the total number of electrons but instead increases the wake acceleration.

The spectra for the cone capillary depend on the laser pointing stability as well as on the ambient gas. When the 

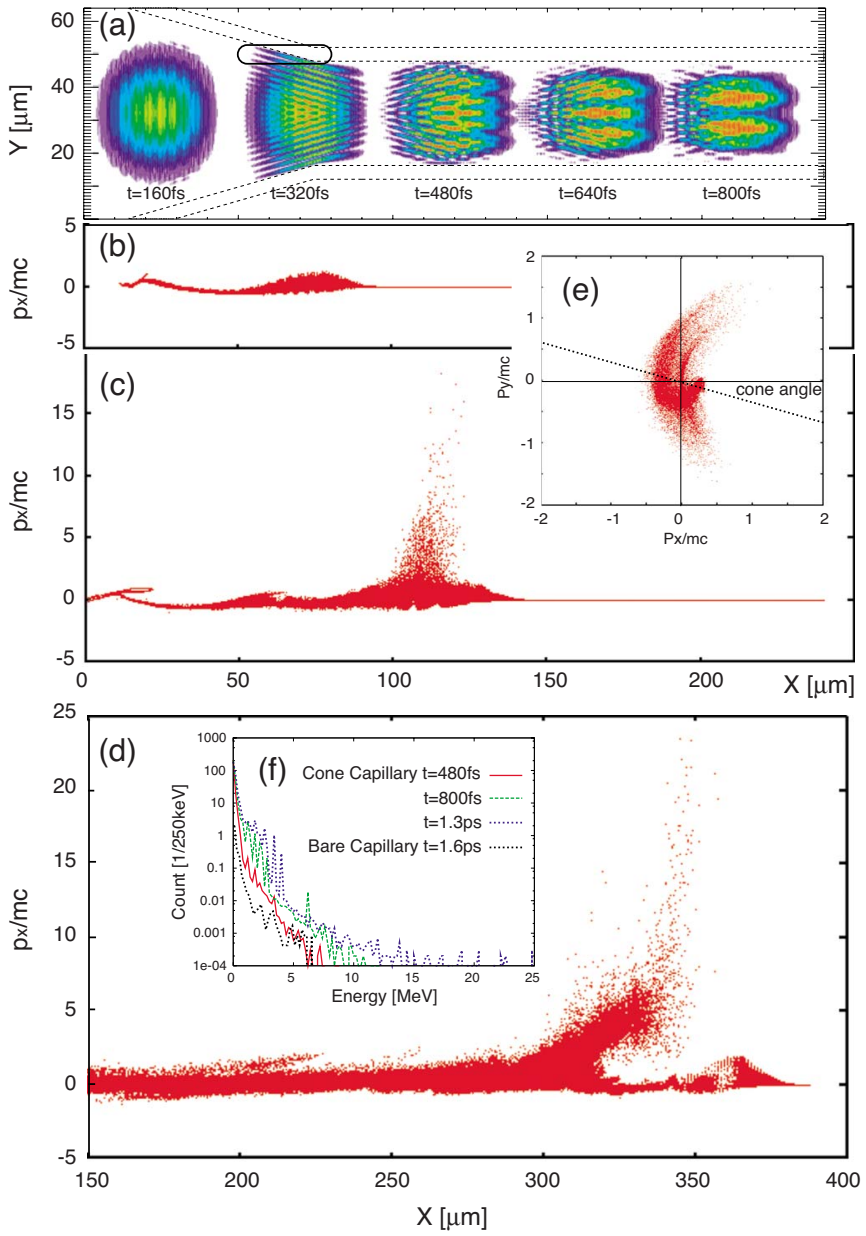

FIG. 5. (Color online) (a) Contour plots of the laser intensity at sequential times. Electron phase plots of $x-p_{x}$ (b) at $320 \mathrm{fs}$ (when the pulse enters the capillary), (c) at $480 \mathrm{fs}$, and (d) at $1.3 \mathrm{ps}$. (e) The electron angular distribution at $320 \mathrm{fs}$ observed near the entrance of the capillary [the region marked by an oval in panel (a)]. (f) Electron energy spectra.

laser hits the inside of the cone surface, the x-ray pinhole camera detects strong $\gamma$-rays. The electron spectra from all three ESMs show that the electrons have isotropic energy distributions below $15 \mathrm{MeV}$. Protons up to $5 \mathrm{MeV}$ were detected using the same ESM. These protons are generated by a direct interaction between the laser beam and a solid target because they were not observed when the beam was correctly aligned. Although electrons up to $15 \mathrm{MeV}$ were observed by all three ESMs, electrons above $15 \mathrm{MeV}$ were only detected by the on-axis spectrometer, indicating that the beam was collimated. In the case of the cone capillary, when the beam was not aligned, electrons above $15 \mathrm{MeV}$ were not detected along the axis.

The electron energy spectra from the three targets indicate that an attached hollow cone-produced relativistic electrons below $10 \mathrm{MeV}$ and that the capillary accelerated these electrons to $30 \mathrm{MeV}$ with a charge of $1.1 \mathrm{pC}$. The energetic electrons were extracted through a cone-laser interaction and automatically accelerated by a wake field driven by the laser propagating inside the capillary. This occurred when the electron density was adjusted to match the wake excitation condition.

\section{WAKE AMPLITUDE AND ELECTRON TRAPPING THRESHOLD}

Experiments reveal the presence of a sideband around $\omega / \omega_{0}=1.006$ with bandwidth $\Delta\left(\omega / \omega_{0}\right)=0.005$, where $\omega / \omega_{0}$ is the emitted frequency normalized to the incident laser frequency. The intensity of the sideband ratio to that of the pump laser $P_{s} / P_{0}$ was 0.05 . One dimensional (1D)-PIC reproduced the experiments for a $2 \mathrm{~cm}$ uniform plasma with $n_{0}=7 \times 10^{15} \mathrm{~cm}^{-3}, \quad \omega / \omega_{0}=1.007, \quad \Delta\left(\omega / \omega_{0}\right)=0.005, \quad$ and $P_{s} / P_{0}=0.04$. The wake amplitude $\epsilon=\delta n / n_{0}$ can be evaluated from the formula $P_{s} / P_{0}=\left[(\pi / 2) \epsilon\left(n_{0} / n_{c}\right)(L / \lambda)\right]^{2},{ }^{24}$ where $\delta n$ is the electron density perturbation, $n_{0}$ is the electron density, $n_{c}$ is the cutoff density, and $L$ and $\lambda$ are the length of the excited plasma wave and the laser wavelength, respectively. Here $\epsilon$ was 0.9 for the plasma with a density of 7 $\times 10^{15} \mathrm{~cm}^{-3}$ assuming it is uniform. From the 1D-PIC, the wake field amplitude was $2 \mathrm{GV} / \mathrm{m}$ and $\epsilon$ was 0.25 . This amplitude results in an energy gain of $40 \mathrm{MeV}$ for a $2 \mathrm{~cm}$ acceleration length. Both simulation and experiments indicate that a wake field with moderate amplitude $\epsilon>0.2$ was excited in the plasma.

A wake field with $\gamma_{p}=\sqrt{n_{c} / n_{0}}$ can trap and accelerate injected electrons having a minimum kinetic energy of $W_{\min }=m c^{2}\left[\gamma_{p}\left(\gamma_{p} \epsilon+1\right)\left(1-\beta_{p} \beta^{\prime}\right)-1\right]$, where $\beta_{p}=\left(1-1 / \gamma_{p}^{2}\right)^{1 / 2}$ and $\beta^{\prime}=\left[1-1 /\left(\gamma_{p} \epsilon+1\right)^{2}\right]^{1 / 2} \cdot{ }^{10,25}$ Trapping of $10 \mathrm{MeV}$ electrons by the wake field at a density of $10^{16} \mathrm{~cm}^{-3}\left(\gamma_{p}=316\right)$ requires a threshold value of $\epsilon=0.021$. The estimated wake amplitude from experiments and the 1D-PIC was well beyond this threshold: $\epsilon \sim 0.2$. Therefore cone-produced electrons can be trapped by the wake field excited in the capillary containing ambient gas.

\section{2D SIMULATION FOR INJECTION OF ELECTRONS INTO WAKE FIELD}

The cone capillary produced an energetic electron beam in the wake field that was not observed for the capillary without an attached cone. The wake field in the cone capillary with ambient gas sustained enough amplitude to trap the electrons produced at the cone surface. In order to investigate the electron-injecting mechanism and the laser wake field trapping, we constructed a two dimensional (2D)-PIC simulation. The simulation conditions were $1 / 5$ scale of the experimental capillary (i.e., an inner diameter of $30 \mu \mathrm{m}$ ) and a pulse length of 160 fs at a plasma density of 2.5 $\times 10^{17} \mathrm{~cm}^{-3}$ and a laser amplitude of $a_{0}=1.5$. The density was chosen so that the ratio of the pulse length to the plasma wavelength was similar to that of the experiment. The initial target shape is shown in Fig. 5. The target consists of a $10 n_{c}$ plasma dense enough to stop and guide the laser pulse. The target density is not critical in this simulation since the pulse interacts with a small preformed plasma that has a scale length of $\sim 1 \mu \mathrm{m}$, located in the interior of the target. This preplasma was based on the experimental conditions. The simulation was run for 1.3 ps so that the pulse propagated about $400 \mu \mathrm{m}$.

The plasma density in the 2D simulation is higher than that in the experiments. However, as far as electron trapping from the cone surface is concerned, the simulation demon- 
strates the correct dynamics. To trap $10 \mathrm{MeV}$ electrons, the wake amplitude threshold is 0.01 for a plasma density of $2.5 \times 10^{17} \mathrm{~cm}^{-3}\left(\gamma_{p}=63\right)$. This threshold is half that in the experiments so that the $2 \mathrm{D}$ simulation might trap electrons of lower energy than did the experiments. However, the experimental wake amplitude is more than an order of magnitude higher than the threshold value, and thus there should be no qualitative difference between experiments and the 2D simulations.

Figure 5(a) shows contour maps of the laser radiation propagating along the cone capillary. They demonstrate that the pulse is introduced into the capillary by optically guiding in the cone and that it propagates stably, although some modulation appears due to interference between the central pulse and the light reflected from the capillary walls. Figures 5(b)-5(d) plot the electron longitudinal phase at three different times in the central region of the capillary. At 320 fs there are no trapped electrons in the wake field excited behind the pulse, although electrons inside the pulse oscillate with a $J \times B$ magnetic force. This oscillation is not sufficiently large to inject electrons in the trapping phase at the given laser intensity. Trapped electrons first appear around $480 \mathrm{fs}$, and they receive a strong impulse from the wake field, indicating that some interaction occurs between the wake field in the capillary and the trapped electrons. It is known that hot electrons are guided along the cone surface by self-generated magnetic and electrostatic fields. ${ }^{26}$ Also some hot electrons are extracted from the surface and ejected at angles defined by the specular direction to the target normal. ${ }^{27}$ The angular distribution of hot electrons around the entrance of the cone target is graphed in Fig. 5(e). It shows that hot electrons are extracted from the cone surface and propagate toward the capillary center. These electrons are automatically injected into the wake field behind the laser pulse and become trapped, as Fig. 5(c) shows. Both Figs. 5(b) and 5(e) show the electron momentum at $320 \mathrm{fs}$. In panel (b), the data include the $x$-component of the momentum of the central region of the capillary. It does not include any electrons ejected from the cone surface shown in panel (e). Subsequently the electrons are accelerated and gain energy until $t=1.3 \mathrm{ps}$. Figure 5(f) shows the electron spectra for different laser pulse arrival locations, namely, (I) the cone exit, (II) after $200 \mu \mathrm{m}$ of propagation, and (III) after $400 \mu \mathrm{m}$ of propagation along the cone capillary, as well as (IV) after $460 \mu \mathrm{m}$ of propagation through the bare capillary.

Figure 5 indicates that the number of high-energy electrons increases as the laser pulse propagates along the capillary. When $t=480 \mathrm{fs}$, the pulse is enclosed in the capillary, and two temperature components can be distinguished in the spectrum. The experimental spectrum of the cone target shown in Fig. 4 also has two temperature components. As the laser propagates along the capillary, high-energy electrons appears at $t>800 \mathrm{fs}$. These are generated by the wake field in the capillary. In Fig. 5(f) for a bare capillary, although the electrons are accelerated, the number of electrons trapped in the wake field is small compared to the cone capillary case. Furthermore the energy gain is low. The injection mechanism here is thus different from that simulated in Ref. 28 for a plasma channel wall. The experimental electron spectrum of the cone capillary has energy components above $5 \mathrm{MeV}$, clearly distinguished from that of the cone target spectrum. Since the dephasing length is much longer than the capillary length at a density of $10^{16} \mathrm{~cm}^{-3}$, the spectrum has a Maxwellian distribution. However, electron trapping by the wake field at low electron densities $\left(<10^{16} \mathrm{~cm}^{-3}\right)$ can occur. These 2D-PIC results support the proposed automatic electron-seeding mechanism for the cone in the wake field.

\section{DISCUSSION}

We have observed electron signal gains when the hollow cone was attached to the entrance of the capillary. Energies of up to $30 \mathrm{MeV}$ are obtained when the capillary is filled with gas and the electron density matches the wake excitation conditions for carefully adjusted laser pointing. The observed increase in the number and energy of the electrons is likely due to autoinjection into the wake of a population of electrons generated in the cone.

On the other hand, the attached cone may enhance the coupling of the laser into the capillary and hence drive a wake in it so that self-trapping leads to the observed signal. The energy threshold for self-trapping depends on the wake field potential and laser ponderomotive force inside the capillary. Such an enhancement will occur if there are significant improvements in the laser intensity and wake potential compared to those in a glass capillary. However, according to the 2D-PIC simulations for the experimental geometries (with the laser spot size smaller than the capillary diameter), there is no significant increase in the laser intensity when the cone is attached and hence no rise in the wake potential and ponderomotive force. Such phenomena would only tend to occur for perfect matching between the laser and cone ${ }^{26}$ of either the cone angle or of the laser spot size and capillary diameter.

Another possibility is that since the light field inside the capillary is multimode (because the spot size is smaller than the capillary diameter), there could be beating between the modes, which could lead to self-trapping at lower amplitudes than might otherwise be expected. However, the modes guided in the glass capillary strongly depend on its geometry and are not influenced by the presence of the cone, particularly in our experimental geometries.

In the simulation, the electrons trapped and accelerated to high energy are initially located near the surface of the cone at the entrance to the capillary [as marked in Fig. 5(a)]. Based on Fig. 5(e), electrons are automatically injected from the cone wall into the entrance of the capillary. When we integrate the total beam charge between 4 and $30 \mathrm{MeV}$ from Fig. 4, an increase in the charge is found in the presence of the cone capillary containing ambient gas compared to the other cases. If injection is due to electrons produced at the cone surface, the charge would remain constant and not increase when gas is introduced. We hypothesize that the increase is instead due to the acceleration of electrons initially below $4 \mathrm{MeV}$. Since the electron spectrum has a Maxwellian distribution, the total beam charge includes low-energy electrons not measured by the ESM. 
To summarize, the experiments show that the hollow cone attached to the entrance of the capillary contributes significant gains in both the number and energy of the electrons. There are two possible explanations for the observed signals between 10 and $30 \mathrm{MeV}$ for the cone capillary containing ambient gas. First, cone-produced electrons may be automatically trapped by the wake field. Second, the cone may enhance the coupling of the laser into the capillary and hence drive the wake, with self-trapping leading to the observed electron signals. Based on the experiments, we cannot conclusively establish which mechanism is dominant. However, the 2D-PIC simulations reveal that cone-produced electrons are automatically trapped and accelerated by the wake field.

\section{CONCLUSIONS}

The hollow cone acts as an automatic injector of highenergy electrons. This is key for low-density capillary electron accelerators. Without the cone, the capillary in the ambient gas generates a collimated electron beam of insufficient energy to be trapped by the wake field. By using the cone capillary, the electrons are accelerated up to $30 \mathrm{MeV}$. This acceleration is due to the laser wake field excited in the capillary. There are two possible explanations for the observed increase in the number of electrons. The first is that the cone extracts electrons, and the second is that the cone enhances the laser coupling into the capillary and the self-trapping. The 2D-PIC simulation is consistent with the first possibility. Auto-electron-injection by cone-laser interactions is an alternative method for wave breaking and optical injection, as has been applied in many recent laser electron acceleration studies. These mechanisms provide a compact electron injector for multi-GeV accelerators.

\section{ACKNOWLEDGMENTS}

The authors thank the staff of the ILE Laser and Target Groups for their assistance during the FA-03 and FB-03 experiments.

This study was supported by the Budget for Advanced Compact Accelerator Development of the Ministry of Education, Culture, Sports, Science, and Technology (MEXT 2001-2006) and a Grant-in Aid for Scientific Research (B) Grant No. 16340182 of MEXT. This work is supported by the research collaboration of the ILE. Y.S. was supported by UNR under DOE/NNSA Grant No. DE-FC52-06NA27616.

${ }^{1}$ T. Tajima and J. M. Dawson, Phys. Rev. Lett. 43, 267 (1979).

${ }^{2}$ Y. Kitagawa, Y. Sentoku, S. Akamatsu, W. Sakamoto, R. Kodama, K. A. Tanaka, K. Azumi, T. Norimatsu, T. Matuoka, H. Fujita, and H. Yoshida, Phys. Rev. Lett. 92, 205002 (2004).

${ }^{3}$ S. P. D. Mangles, C. D. Murphy, Z. Najmudin, A. G. R. Thomas, J. L. Collier, A. E. Dangor, E. J. Divall, P. S. Foster, J. G. Gallacher, C. J.
Hooker, D. A. Jaroszynski, A. J. Langley, W. B. Mori, P. A. Norreys, F. S. Tsung, R. Viskup, B. R. Walton, and K. Krushelnick, Nature (London) 431, 535 (2004).

${ }^{4}$ C. G. R. Geddes, Cs. Tóth, J. van Tilborg, E. Esarey, C. B. Schroeder, D. Bruhwiler, C. Nieter, J. Cary, and W. P. Leemans, Nature (London) 431, 538 (2004)

${ }^{5}$ J. Faure, Y. Glinec, A. Pukhov, S. Kiselev, S. Gordienko, E. Lefebvre, J.-P. Rousseau, F. Burgy, and V. Malka, Nature (London) 431, 541 (2004).

${ }^{6}$ E. Miura, K. Koyama, S. Kato, N. Saito, M. Adach, Y. Kawada, T. Nakamura, and M. Tanimoto, Appl. Phys. Lett. 86, 251501 (2005).

${ }^{7}$ M. Mori, M. Kando, I. Daito, H. Kotaki, Y. Hayashi, A. Yamazaki, K. Ogura, A. Sagisaka, J. Koga, K. Nakajima, H. Daido, S. V. Bulanov, and T. Kimura, Phys. Lett. A 356, 146 (2006).

${ }^{8}$ W. P. Leemans, B. Nagler, A. J. Gonsalves, Cs. Tóth, K. Nakamura, C. G. R. Geddes, E. Esarey, C. B. Schroeder, and S. M. Hooker, Nat. Phys. 2, 696 (2006).

${ }^{9}$ K. Nakamura, B. Nagler, Cs. Tóth, C. G. R. Geddes, C. B. Schroeder, E. Esarey, A. J. Gonsalves, S. M. Hooker, and W. P. Leemans, Phys. Plasmas 14, 056708 (2007).

${ }^{10}$ Y. Kitagawa, T. Matsumoto, T. Minamihata, K. Sawai, K. Matsuo, K. Mima, K. Nishihara, H. Azechi, K. A. Tanaka, H. Takabe, and S. Nakai, Phys. Rev. Lett. 68, 48 (1992).

${ }^{11}$ C. E. Clayton, K. A. Marsh, A. Dyson, M. Everett, A. Lal, W. P. Leemans, R. Williams, and C. Joshi, Phys. Rev. Lett. 70, 37 (1993).

${ }^{12}$ F. Amiranoff, S. Baton, D. Bernard, B. Cros, D. Descamps, F. Dorchies, F. Jacquet, V. Malka, J. R. Marqués, G. Matthieussent, P. Miné, A. Modena, P. Mora, J. Morillo, and Z. Najmudin, Phys. Rev. Lett. 81, 995 (1998).

${ }^{13}$ D. Umstadter, J. K. Kim, and E. Dodd, Phys. Rev. Lett. 76, 2073 (1996).

${ }^{14}$ E. Esarey, P. Sprangle, J. Krall, and A. Ting, IEEE Trans. Plasma Sci. 24, 252 (1996)

${ }^{15}$ G. Fubiani, E. Esarey, C. B. Schroeder, and W. P. Leemans, Phys. Rev. E 70, 016402 (2004).

${ }^{16}$ H. Kotaki, S. Masuda, M. Kando, J. K. Koga, and K. Nakajima, Phys. Plasmas 11, 3296 (2004).

${ }^{17}$ J. Faure, C. Rechatin, A. Norlin, A. Lifschitz, Y. Glinec, and V. Malka, Nature (London) 444, 737 (2006).

${ }^{18}$ A. Zhidkov, J. Koga, K. Kinoshita, and M. Uesaka, Phys. Rev. E 69, 035401(R) (2004).

${ }^{19}$ Y. Kitagawa, H. Fujita, R. Kodama, H. Yoshida, S. Matsuo, T. Jitsuno, T. Kawasaki, H. Kitamura, T. Kanabe, S. Sakabe, K. Shigemori, N. Miyanaga, and Y. Izawa, IEEE J. Quantum Electron. 40, 281 (2004).

${ }^{20}$ K. A. Tanaka, T. Yabuuchi, T. Sato, R. Kodama, Y. Kitagawa, T. Takahashi, and S. Okuda, Rev. Sci. Instrum. 76, 013507 (2005).

${ }^{21}$ T. Yabuuchi, K. Adumi, H. Habara, R. Kodama, K. Kondo, T. Tanimoto, K. A. Tanaka, Y. Sentoku, T. Matsuoka, Z. L. Chen, M. Tampo, A. L. Lei, and K. Mima, Phys. Plasmas 14, 040706 (2007).

${ }^{22}$ Y. Kitagawa, R. Kodama, K. Takahashi, M. Mori, M. Iwata, S. Tsuji, K. Suzuki, K. Sawai, K. Hamada, K. A. Tanaka, H. Fujita, T. Kanabe, H. Takabe, H. Habara, Y. Kato, and K. Mima, Fusion Eng. Des. 44, 261 (1999).

${ }^{23}$ M. V. Ammosov, N. B. Delone, and V. P. Krainov, Sov. Phys. JETP 64, 1191 (1986)

${ }^{24}$ R. E. Slusher and C. M. Surko, Phys. Fluids 23, 472 (1980).

${ }^{25}$ N. A. Ebrahim and S. R. Douglas, Laser Part. Beams 13, 147 (1995).

${ }^{26}$ Y. Sentoku, K. Mima, H. Ruhl, Y. Toyama, R. Kodama, and T. E. Cowan, Phys. Plasmas 11, 3083 (2004).

${ }^{27}$ Y. Sentoku, H. Ruhl, K. Mima, R. Kodama, K. A. Tanaka, and Y. Kishimoto, Phys. Plasmas 6, 2855 (1999).

${ }^{28}$ F. S. Tsung, R. Narang, W. B. Mori, C. Joshi, R. A. Fonseca, and L. O. Silva, Phys. Rev. Lett. 93, 185002 (2004). 